\title{
Labyrinthe
}

32 | 2009 (1)

Le petit théâtre intellectuel

\section{Laibach, ou Le Collectif totalitaire}

\section{Laurent Dubreuil}

\section{OpenEdition}

\section{Journals}

\section{Édition électronique}

URL : http://journals.openedition.org/labyrinthe/3999

DOI : 10.4000/labyrinthe.3999

ISSN : 1950-6031

Éditeur

Hermann

\section{Édition imprimée}

Date de publication : 19 juin 2009

Pagination : 99-102

ISBN : 978-2-7056-6885-3

\section{Référence électronique}

Laurent Dubreuil, «Laibach, ou Le Collectif totalitaire », Labyrinthe [En ligne], 32 | 2009 (1), mis en ligne le 01 février 2011, consulté le 21 avril 2019. URL : http://journals.openedition.org/labyrinthe/3999 ; DOI : 10.4000/labyrinthe.3999 


\section{LAIBACH, OU LE COLLECTIF TOTALITAIRE}

Depuis 1980, « Laibach » nomme une tentative artistique et théorique. Laibach est un groupe de rock fondé à Ljubljana ${ }^{1}$ qui a su emprunter à des formes d'expérimentation musicale, à l'imagerie politique des régimes totalitaires, à la posture avant-gardiste, performance intellectuelle incluse. Dans la première décennie, ce qui parvenait en Europe occidentale du mouvement Neue slowenische Kunst (ou NSK, nouvel art slovène) et de son plus glorieux représentant était lacunaire. Ce qui, en un sens, tombait bien, car malgré les affirmations unitaristes du collectif Laibach, l'œuvre et la réflexion produites se dispersaient toujours en diverses directions. Donc à cette époque reculée qui pré-existait au web et à la chute du mur de Berlin, Laibach existait à l'Ouest par ses disques, parfois introuvables, mais dont la plupart étaient pressés par Mute Records ${ }^{2}$. On y entendait des voix chanter ou déclamer en slovène, en anglais, en allemand. Des orchestrations pompeuses, pleines de cors post-wagnériens, de chœurs post-straussiens. Une trame bruitiste, surtout dans les débuts, qui caractérisait la première insertion dans les alentours du rock industriel - les pochettes de disques laissaient penser que les membres du groupe avaient un certain goût pour l'habit traditionnel des Alpes germaniques, le cuir, les uniformes vert-de-gris et les complets rouille. Des citations de leaders staliniens, comme Walter Ulbricht, voisinaient avec des clichés de spectacle rappelant visuellement les hautes heures du Nuremberg nazi ${ }^{3}$. Deux traits distinctifs de cette Laibachmusik à la fin des années 1980 semblaient le recours massif à un gros son, exagéré, qu'il soit hard rock amplifié ou appuis symphoniques - et un art de la cover, qui conduisait au consciencieux dynamitage de l'original. Let It $B e^{4}$, la reprise quasi

\footnotetext{
1. Pour la plus grande part de son histoire, la ville de Ljubljana (ou Laibach en allemand) a appartenu aux Habsbourg, et donc à l'empire d'Autriche.

2. Label spécialisé dans la musique synthétique et le rock expérimental, où figuraient Depeche Mode, Einstürzende Neubauten, Diamanda Galas ou Nick Cave.

3. En particulier Laibach, Krst pod triglavom, Sub Rosa, 1987.

4. Laibach, Let It Be, Mute Records, 1988.
} 
intégrale de l'album des Beatles sorti sous le même titre, est le chefd'œuvre du genre où la gentille pop du quatuor britannique est pulvérisée. Il n'échappait pas à grand monde, donc, même hors concert et même depuis une perspective morcelée, que Laibach réarrangeait le pré-établi, en laissant passer des éléments hétérogènes, voire nauséabonds. Le sens, lui, était très douteux. « Perspectives », une ancienne chanson-manifeste ${ }^{1}$, avertissait pourtant longuement les auditeurs qu'il ne s'agissait pas que de provocation rigolote. La voix impeccablement sérieuse préconisait, par les moyens de l'art, une « forme de terreur systématique, physique et psychique, organisées conformément aux relations sociales. » Plus : « Notre œuvre est industrielle, notre langage politique. [...] L'approche musicale de Laibach consiste à aller vers une zone de pure politisation du son qui soit un moyen de manipuler les masses. »

Au fur et à mesure que ce travail devenait plus largement accessible par la multiplication de canaux médiatiques, par la levée du rideau de fer, par l'accroissement de l'œuvre même, fut alors connu ce qui formait jusque-là une évidence pour les seuls Yougoslaves ou à peu près : Laibach articulait un discours «d'intellectuels » sur la société, le pouvoir, le contemporain. L'humour un peu épais existait bel et bien, celui qui présidait à la transposition ralentie et germanisée de l'inepte « Live Is Life » (du groupe Opus) dont les « nanananana » étaient remplacées par un trio d'orgue, cor et guitare électrique. Il existait, mais il n'était pas une fin. Quelles que fussent les potentialités parodiques de sa musique, Laibach, pris dans l'ensemble NSK, avec toute son imagerie ${ }^{2}$, soudain accessible et presque toujours inspirée du stalinisme et du nazisme, disait plus que la dérision. Laibach passait de la disco (« le moyen le plus approprié de manipulation médiatique ») à l'indus à la techno au symphonique, de la reprise des Beatles à celle de l'esthétique du Troisième Reich, selon une logique apparentée au détournement qu'a théorisé Guy Debord ${ }^{3}$. La condamnation des « deux » modèles de société, dans Laibach comme le situationnisme, mène à un semblable recours aux artefacts de la culture de masse, dans l'espoir réputé avant-gardiste de leur faire cracher leur vérité d'organisation, et, peut-être, de les subvertir. Bien sûr, le corps

\footnotetext{
1. À trouver dans Laibach, Rekapitulacija 1980-1984, NSK Recordings, 2002.

2. Pour les amateurs, l'ouvrage d'Alexei Monroe, Interrogation Machine. Laibach and NSK, Cambridge (États-Unis), The MIT Press, 2005 donne une idée plus complète des activités de NSK.

3. En particulier Guy Debord, La Société du spectacle, § 208.
} 
de doctrine de Laibach est resté à la fois moins volumineux et, surtout, ésotérique, rejoignant en cela plus les sectes organisées par un Gourou que les modèles de confréries artistiques et politiques rêvées par Debord. Assurément, alors qu'une dimension nouvelle est révélée par l'appariement d'un extrait de western et d'un commentaire serré de Marx dans le film La Société du spectacle, Laibach montrait une connivence étrange en se contentant de traduire en allemand les paroles de « One Vision » du groupe Queen et de re-baptiser le tout « Geburt einer Nation » (« Naissance d'une nation »). Laibach agissait comme révélateur des affinités autoritaires entre le wagnérisme politique et l'entertainment.

Ainsi la collation et le collage de signes (brassard à croix crantée, rythme techno, moustaches et bretelles, lyrics de chansons à succès, évocation de concepts, etc.) servent une entreprise pythique. Laibach dévoile. La société de masse - consumériste, nazie ou communiste, peu importe - est une dictature. Il existe en commun un apparat, dont l'aspect totalitaire est justement révélé par la capacité de migration des signes. Pour exhiber cela, car il s'agit bien plus pour Laibach de montrer que de démontrer, la stratégie utilisée est une forme de saturation totalitaire. Comme la prophétesse, les membres de Laibach sont proprement intoxiqués. Mithridatisés ? j'en doute. Slavoj Žižek, qui voit juste en contestant que Laibach se place sur un simple niveau ironique, parle de «sur-identification » au pire du pouvoir, et qui en annulerait alors l'efficacité ${ }^{1}$. Or je crains que la plupart des admirateurs du groupe soient positivement fascinés par la force totalitaire de ces œuvres. Plus. Contrairement à la suggestion de Žižek sur « l'indécidabilité »², et selon laquelle « Laibach et NSK » ne sont pas plus fascistes que non-fascistes, le système de surenchère induit un sérieux de la pose. Laibach et NSK doivent être fascistes, et nazis, et antisémites, et totalitaires, etc., jusqu'au bout des ongles, avec une insistance et une naïveté dont l'absolu permet le dessein. Il ne serait pas possible de formuler un jugement sur le devenir-totalitaire de la prescription politique via l'imagerie de Laibach si celle-ci n'était qu'adventice ou même suspensive. Non. Aucune distance du tout. Laibach est aussi immonde qu'il y paraît, sans quoi le dispositif

\footnotetext{
1. Slavoj Žižek, « Why are Laibach and NSK not Fascists? » (1993), http://www.nskstate.com/appendix/ articles/why_are_laibach. php, consulté le 13 janvier 2009. Ce serait à mettre en rapport avec l'essai de surfascisme chez Georges Bataille, à l'époque du Collège de sociologie.

2. Ibidem.
} 
général ne fonctionne pas et nous en restons à une vague convocation de clichés choc sans liens entre eux, ou à une bonne grosse rigolade de droite nationale. Laibach est en quelque sorte l' ' intellectuel » symbolique, ou en deux pièces : il faut la participation de l'auditeur pour qu'une parole ait lieu. Non au sens phénoménologique, qui serait trivial, mais structurellement. Laibach est neutralisé, annulé par sa propre saturation de citations référentielles; l'acte intellectuel tient dans la constitution de l'énigme (le groupe) et sa résolution (le public). Le «message » est creux; nous le remplissons. Là, sur un mode mineur par rapport aux grandes ambitions apocalyptiques sur l'ordre mondial, Laibach est enfin, et involontairement, le Témoin hystérisé d'un état contemporain de «l'intellectuel » paré à toutes les variations du sens pourvu que soit reconnu son statut, un Renégat perpétuel et prêt à l'emploi ${ }^{1}$.

L. D.

1. D'où la possibilité, pour le groupe, de soudain réinterpréter Jean-Sébastien Bach au synthétiseur et de faire passer cela pour une grande performance d'art con (Laibach, Kunst der Fuge, Mute, 2008) quand il s'agit surtout d'une facile actualisation de la transposition sur un Moog par Walter Carlos, Switched-on Bach, Columbia Records, 1968. Laibach peut renier jusqu'à l'ambition de Laibach pour continuer son parcours. 